\title{
Design of bidirectional frictional behaviour for tactile contact using ellipsoidal asperity micro-textures
}

\author{
Dmitrii SERGACHEV ${ }^{1, *}$, David MATTHEWS ${ }^{1}$, Emile VAN DER HEIDE $^{1,2}$ \\ ${ }^{1}$ Laboratory for Surface Technology and Tribology, Faculty of Engineering Technology, University of Twente, Overijssel 7522 LW, \\ Enschede, the Netherlands \\ ${ }^{2}$ Faculty of Engineering and Architecture, Ghent University, East Flanders 9000, Ghent, Belgium \\ Received: 26 January 2021 / Revised: 21 April 2021 / Accepted: 17 May 2021 \\ (C) The author(s) 2021.
}

\begin{abstract}
Tactile perception and friction can be modified by producing a deterministic surface topography. Change of surface feature arrangement and texture symmetry can produce an anisotropic frictional behaviour. It is generally achieved through skin hysteresis by promoting its deformation. This work investigates whether a bidirectional friction can be created with microscale ellipsoidal asperity textures, thus relying on the adhesive component of friction. For this purpose, four textured samples with various asperity dimensions were moulded with a silicone rubber having an elastic modulus comparable to that of the skin. Coefficient of friction measurements were conducted in-vivo in two sliding directions with a range of normal loads up to 4 N. Finite element method (FEM) was used to study elastic deformation effects, explain the observed friction difference, and predict surface material influence. Measurements performed perpendicular to the asperity major radii showed consistently higher friction coefficients than that during parallel sliding. For the larger asperity dimensions, a change of the sliding direction increased friction up to a factor of 2 . The numerical analysis showed that this effect is mostly related to elastic asperity deflection. Bidirectional friction differences can be further controlled by asperity dimensions, spacing, and material properties.
\end{abstract}

Keywords: surface texture; sliding direction; skin friction; elastomer

\section{Introduction}

People interact through touch with numerous objects and rely on perceived surface properties in everyday tasks. An individual's judgement of a surface is commonly described by four perceptual scales: hardness, warmth, roughness, and stickiness [1, 2]. The latter three are directly related to surface topography and, therefore, can be engineered through surface design. However, the relationship between tribological mechanisms and touch perception is not fully understood. There are reported correlations between surface roughness and perceived coarseness [3], as well as between calculated friction coefficients and stickiness [4]. Ndengue et al. [5] show that samples mimicking natural wood are perceived differently depending on the material they are produced from, vinyl or polyurethane resin, even though the topographical features remain identical. Recently Massimiani et al. [6] and Faucheu et al. [7] performed elaborate studies on the connection between perception and surface texture dimensions. Interestingly, the textures with micro-scale features and spacing below $160 \mu \mathrm{m}$ have shown not only the lowest friction but were uniformly categorized by volunteers as smooth and most likeable.

Friction forces acting on a finger pad can be represented as a sum of interfacial adhesion and deformation terms $[8,9]$. The adhesion term remains predominant during sliding against surfaces of various roughness [10-13]. Hysteresis deformation becomes

* Corresponding author: Dmitrii SERGACHEV, E-mail: d.sergachev@utwente.nl 
significant with an addition of macro-scale surface texturing or features with dimensions in the order of millimetres [14, 15]. Tomlinson et al. [11] found an interlocking friction mechanism to become predominant during finger pad sliding over triangular ridges with the heights over $42.5 \mu \mathrm{m}$.

With adhesion being predominant and directly proportional to the real contact area, finger pad friction can be modified by controlling surface roughness $[10,16,17]$, or for example by micro-texturing [18-21]. The first approach generally assumes stochastic roughness, which is relatively easy to manufacture, but its frictional performance is difficult to predict and reproduce due to a featureless topography. On the other hand, texturing allows to design the contact conditions, develop contact models, and estimate frictional behaviour [18, 22, 23].

By introducing a surface texture, frictional behaviour can be changed with respect to the sliding direction. It is generally achieved through the creation of wavy or parallel-ridge textures [14, 24, 25], which utilize skin hysteresis either to reduce the contact area during sliding or to promote deformation. While more complicated textures with anisotropic frictional behaviour can be produced, such as hook-like spines [26] or shark denticle [27], experimental work with a skin has not been performed yet. Moreover, there are no studies on the feasibility of bisymmetric microtextures and their frictional performance, known to the authors.

The aim of the current work is to investigate whether a bidirectional frictional behaviour can be created with a microscale surface texture. This implies that difference in friction is achieved not by the deformation of the finger pad skin, but by changing the contact area.
For this purpose, textures composed of ellipsoidal asperities were produced on a soft silicone rubber with an elastic modulus comparable to that of the skin. The nature of anisotropic frictional behaviour can be explained through the analysis of friction measurements and the aid of the finite element simulations. Microtextures with frictional dependency on sliding direction can possibly be used to direct the tactile interaction or improve the grip in specific direction without making significant changes to a product appearance or its feel.

\section{Materials and methods}

\subsection{Materials}

Four texture designs with square-packed ellipsoidal asperities were used for this study. A lateral spacing of $200 \mu \mathrm{m}$ was kept for all the samples, while ellipsoid dimensions varied. All ellipsoidal textures in this study have the same minor asperity radius of $25 \mu \mathrm{m}$ and two major radii of 50 and $75 \mu \mathrm{m}$ (Fig. 1). Each of those designs were produced with the heights of 30 and $50 \mu \mathrm{m}$. The samples are referred in the text by their major asperity length as E100 and E150 and by their height as $\mathrm{H} 30$ and $\mathrm{H} 50$, respectively. The surface topography was obtained using S neox 3D Optical Profiler (Sensofar, Spain).

Stainless steel was laser textured to produce negative texture designs, which were used for moulding of silicone rubber master samples. In turn, master samples were replicated by double moulding technique, using polyurethane resin Smooth-Cast ${ }^{\circledR} 305$ (Smooth-on, USA) for a negative intermediate mould. This step was introduced to reduce adhesion during demoulding
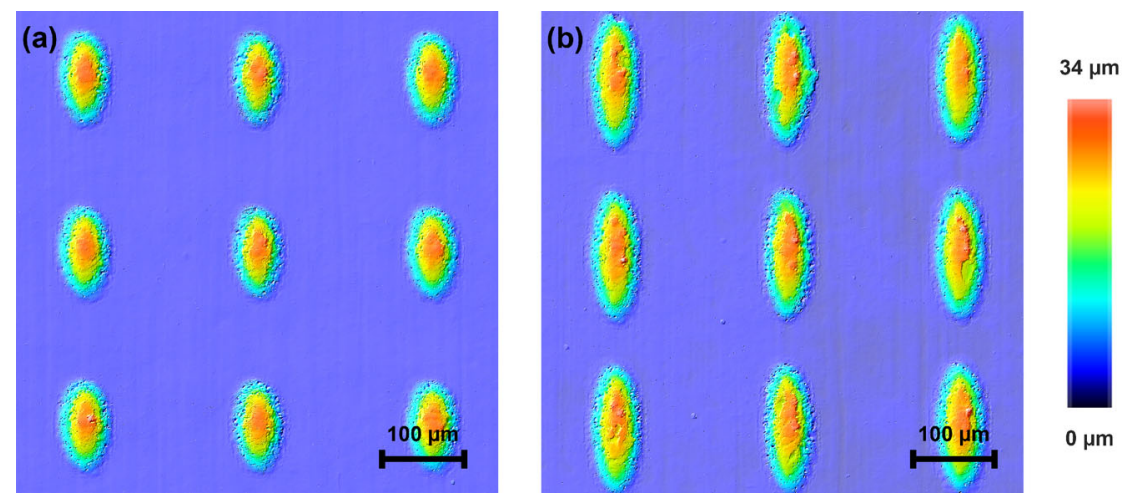

Fig. 1 Ellipsoidal asperity textures with major radius of (a) $50 \mu \mathrm{m}$ and (b) $75 \mu \mathrm{m}$. 
process and improve microtexture reproducibility. Specimens with a thickness of $10 \mathrm{~mm}$ were produced using commercial silicone rubber Mold Max ${ }^{\mathrm{TM}}$ MM10 (Smooth-on, USA), which has an approximate elastic modulus of $0.2 \mathrm{MPa}$. It was selected to promote deformation of elastomer structure over the skin during sliding.

\subsection{Friction measurements}

Friction measurements were performed in-vivo on a setup with a linear reciprocating tribometer depicted on Fig. 2 [28]. A transducer with an attached sample is mounted on a mechanical hinge driven by a spindle. The right hand is placed palm up on a stage below, with an index finger positioned on a 30-degree wedge. The sample is positioned on top of the finger pad and the normal load is applied with static weights.

The friction behaviour between textures and index finger pad was investigated with one subject, one of the authors of this study. The samples were moved from the subject's body with a constant velocity of $2 \mathrm{~mm} / \mathrm{s}$ and an initial acceleration of $2 \mathrm{~mm} / \mathrm{s}^{2}$. The stroke distance was $20 \mathrm{~mm}$. The hinge with the sample was lifted after each measurement and returned to its home position.

Samples were tested with a range of normal loads from 0.2 to $2 \mathrm{~N}$ with a step of $0.2 \mathrm{~N}$ and further up to $4 \mathrm{~N}$ with a step of $0.5 \mathrm{~N}$. Three consecutive measurements were performed with each normal load. Before the start of the measurements 3 preparatory strokes were performed at $0.2 \mathrm{~N}$ load to introduce a sebum layer on a sample surface and ensure consistent conditions across normal loads.

Friction measurements were split into two sets, with texture asperity heights of 30 and $50 \mu \mathrm{m}$,

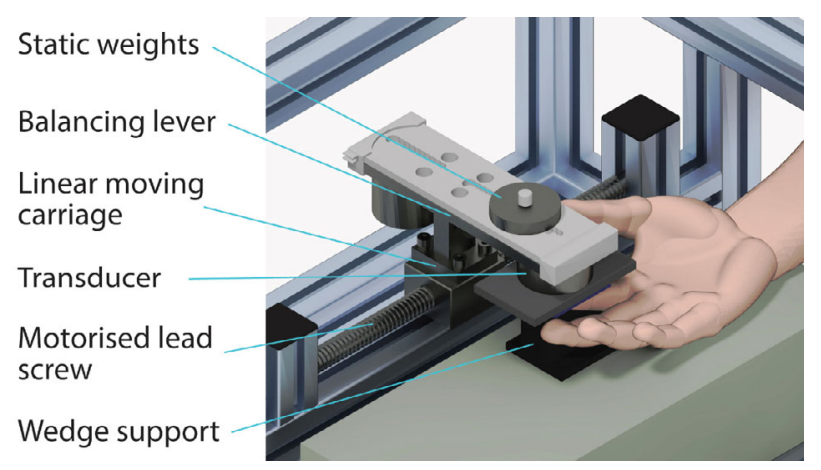

Fig. 2 Schematic representation of the test setup. respectively. Each set consisted of two textured surfaces with different asperity length produced in duplicate and a reference non-textured sample. The measurements were performed in two sliding directions: parallel and perpendicular to the major asperity radii. Each sample set was tested in two different days to confirm that the results are related to texture design and independent of skin properties. This constitutes four measurement series which are denoted as S1 to S4 in the study. The sample order was randomized during each series, while the normal load was increased consequently. Randomization of normal loads was not viable for a chosen approach. After removal of the normal load finger pad takes time to recover to its original shape due to its viscoelastic properties. Therefore, it would either introduce random deviations in friction measurements or significantly increase testing times.

The samples were wipe-cleaned with acetone and alcohol, rinsed with demineralized water, and kept in the vacuum chamber for $12 \mathrm{~h}$ prior to the measurements to prevent rubber swelling.

Experiments were performed in ambient conditions. Average temperature and relative humidity were calculated as $22 \pm 1{ }^{\circ} \mathrm{C}$ and $43 \% \pm 7 \%$ respectively. Hands were washed with soap and air dried for 15 min before each sample measurement. Skin hydration level was checked between the measurements with Corneometer CM 825 (Courage + Khazaka GmbH, Germany). Corneometer values remained stable between days of measurements with a mean of $50 \pm 4$ arbitrary units.

\subsection{Data processing}

A 6-axis Mini40 transducer (Schunk, Germany) was used to measure the forces at $100 \mathrm{~Hz}$ sampling rate. The load cell has a resolution of 6 and $2 \mathrm{mN}$ in the normal and tangential directions, respectively.

The data from the individual measurement strokes was filtered to find the average dynamic friction values for each normal load. A MATLAB code was used to separate the stable region of the curve during sliding from the initial slope, which corresponds to elastic deformation. A typical example of measured signals is shown in Fig. 3. Moreover, a high wavelength noise was induced by the linear driving mechanism, which corresponds to the pitch distance of the shaft and has a constant frequency of $0.5 \mathrm{~Hz}$ with the used 


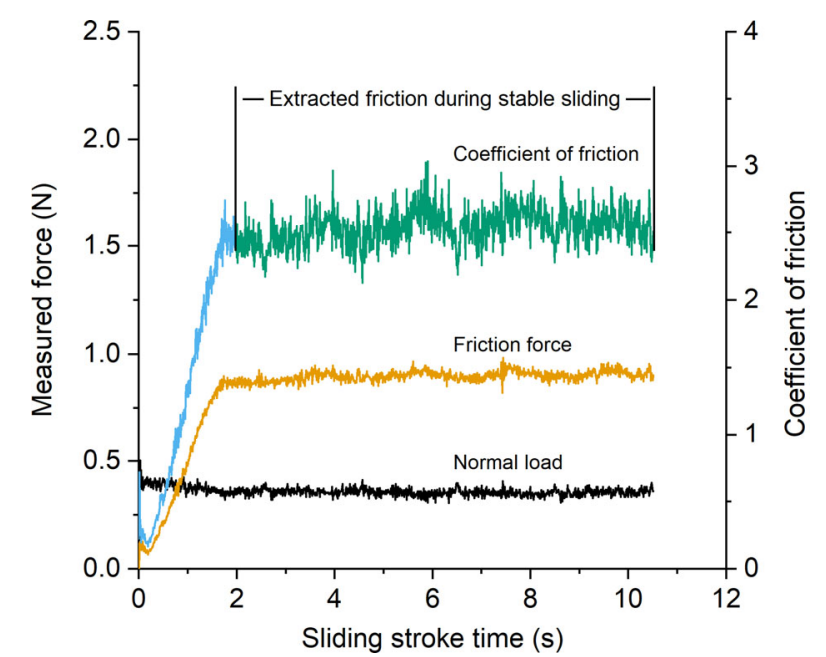

Fig. 3 An example of raw force signals with calculated and extracted dynamic friction coefficient. Data corresponds to a sample E150 $\mathrm{H} 50$ tested in perpendicular direction at $0.4 \mathrm{~N}$ normal load.

sliding velocity. A sine wave fit with a given frequency was determined by a MATLAB code for each measurement to determine a phase offset and an amplitude. The corresponding sine function was subtracted from the original signal to reduce the standard deviation. The calculated average dynamic friction values were not influenced by this operation.

\subsection{Design of computational experiment}

The objective of the finite element method (FEM) analysis was to study contact behaviour of a single asperity under shear and normal loading. A parametric study was performed to characterize the influence of asperity size, Young's modulus, and sliding direction on the contact area development.

The model is represented by two bodies: an asperity and a sliding block, both modelled as isotropic linear elastic elements (Fig. 4). An asperity was meshed with tetrahedral-type elements with an average size of $10 \mu \mathrm{m}$ and distortion control was used to prevent excessive mesh warping. The loading block was meshed with hexahedral-type elements of the same size.

Interaction between two surfaces was defined by penalty friction formulation. To represent an adhesive friction component, which remains predominant in studied contact conditions, the friction coefficient was set to 1 , while the maximum shear stress was limited to $10 \mathrm{kPa}$. Therefore, interfacial shear force remained mostly independent of contact pressure, simulating

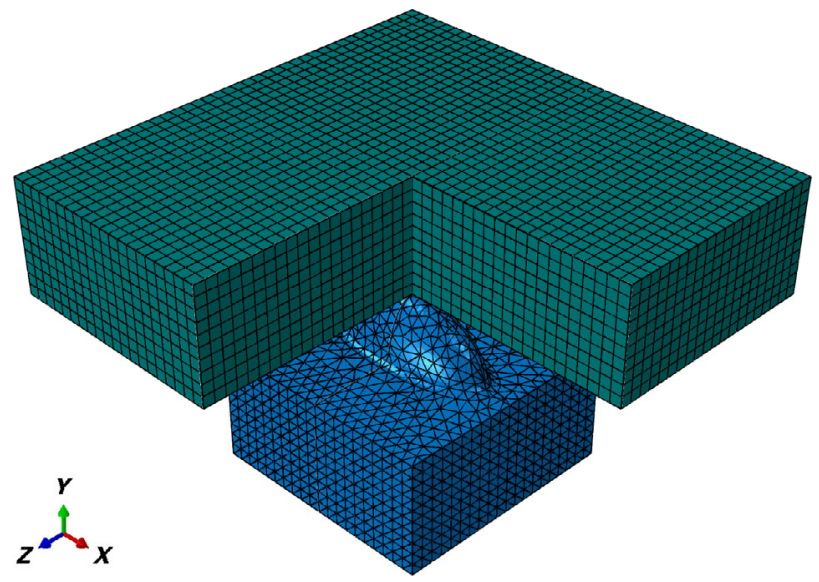

Fig. 4 An example of an element layout and body meshing for E150 H50. Upper block is shown under a section view.

adhesive contact. Surface self-contact for the asperity body was set to frictionless to reduce simulation times.

Initial boundary conditions were specified to restrict body movement. Asperity block was fixed at the bottom plane and its sides were not allowed to move in XZ plane. Each FEM analysis consisted of two calculation steps with prescribed displacement boundary conditions for the upper body:

-Vertical translation to induce normal load;

-Horizontal translation at constant normal displacement which ensured contact sliding.

There was no intention to simulate the mechanical behaviour of the skin with the current FEM study; yet only to represent similar normal displacement. Therefore, the upper body was assumed as an elastic body with Young's modulus of $0.4 \mathrm{MPa}$, which was estimated in our previous study [29]. Asperity properties were set according to the tested material with the elastic modulus of $0.2 \mathrm{MPa}$. Poisson's ratio of 0.49 was used for both bodies [30, 31]. For a continuous sliding contact, inertial forces do not alter solution significantly. Therefore, fixed mass scaling was applied to the entire model to reduce calculation times.

\section{Results and discussion}

\subsection{Friction measurements}

Calculated friction coefficients for the textures with asperity height of $30 \mu \mathrm{m}$ are presented in Fig. 5. The reference surfaces, selected to represent the maximum real area of contact, showed friction coefficient values 

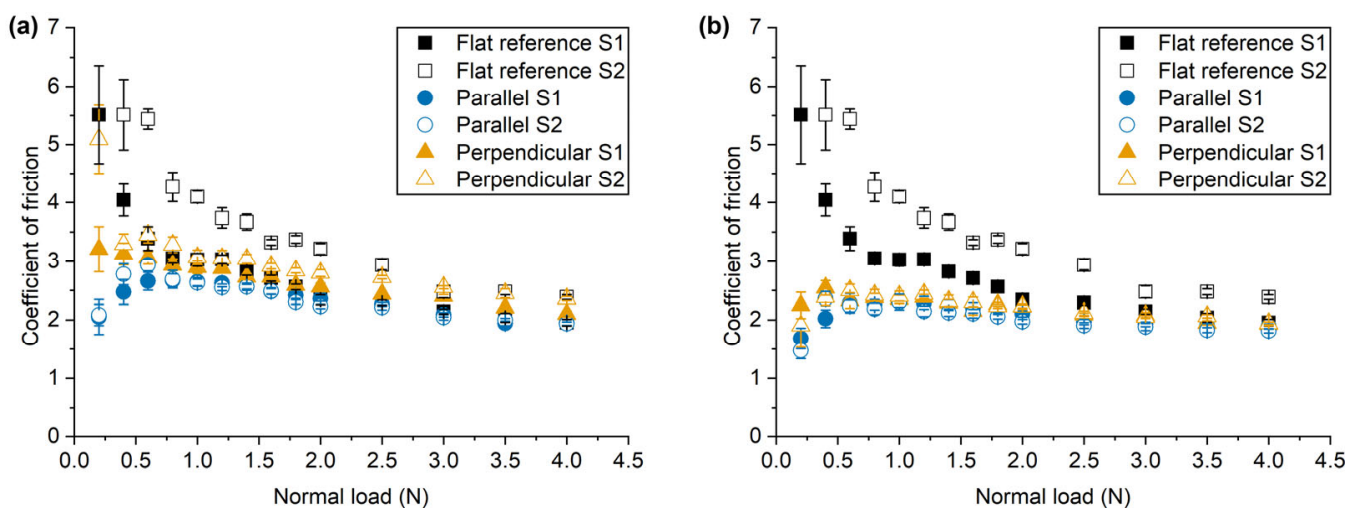

Fig. 5 Coefficient of friction for $30 \mu \mathrm{m}$ height textures (a) E100 and (b) E150. Corresponding measurement series are denoted as $\mathrm{S} 1$ and $\mathrm{S} 2$.

that ranged from about 6.5 at the lower end of the normal force scale down to about 2.5 for the higher end of the scale. All non-textured reference samples follow a power law trend commonly observed for an adhesion-based frictional response. Part of the differences in the resulting coefficient of friction for the reference surfaces might well be due to variation in local interfacial shear stress or day-to-day differences in elastic conditions of the finger pad, yet part was also due to differences in roughness of the reference surfaces. The reference sample tested during the first measurement series showed considerably lower friction coefficient. Its surface roughness average $S_{a}$ was measured around $150 \mathrm{~nm}$, while for other reference samples it was between 40 and $60 \mathrm{~nm}$. Reduction of the friction coefficient with increase of roughness is consistent with literature [10, 16, 17, 19].

Both textured surfaces showed the lowest friction during parallel sliding. It increased with normal load up to a maximum of 2.7 and 2.3 for E100 (Fig. 5(a)) and E150 (Fig. 5(b)), respectively. After reaching these peak values at $1 \mathrm{~N}$ load, the coefficient of friction declines steadily almost reaching the values of the reference sample. Such behaviour is explained by the change in a contact state from asperity to full contact. When sliding is performed perpendicular to the major radii, the coefficient of friction increases slightly for all normal loads tested. The greatest difference between sliding directions is observed at normal loads below $0.5 \mathrm{~N}$ and a local peak is less prominent during perpendicular sliding.

Contact with a textured surface can be represented by one of the following three states. At low normal loads skin is fully supported by asperities of the interfacing surface. The real contact area is limited, which results in lower friction forces. With increase of the applied load surfaces deform, and skin reaches a texture valley. Contact transition increases the rate of contact area growth with normal load and leads to the increase in friction. Finally, the contact stabilizes at full contact and further contact area development is restricted. This effect was described previously by other researchers for grooved $[23,24]$ and bump textures [18].

Increase of the asperity heights from 30 to $50 \mu \mathrm{m}$ predictably led to lower friction coefficients during parallel sliding (Fig. 6). A one-tailed paired t-test was performed on average values of the measurement series paired by normal load. The $p$-values for the t-test were lower than 0.05 for both E100 and E150 textures with $p$-values of $1.2 \mathrm{e}-5$ and 8.6e- 6 respectively, which indicate the results are statistically highly significant. The maximum coefficient of friction values of 2.2 and 2.0 were reached during parallel sliding for E100 and E150, respectively. Textures with $50 \mu \mathrm{m}$ asperity heights have a distinctive contact transition stage. The distinction between parallel and perpendicular sliding also became more prominent. The largest difference is observed at lower normal loads, but it diminishes with an increase of applied normal load.

Significant friction difference at lower loads, while surfaces remain in asperity contact, implies that this behaviour is caused by the contact area change and has a predominantly adhesive nature. It is also supported by the fact, that increase of the asperity heights and lateral dimensions is accompanied with 

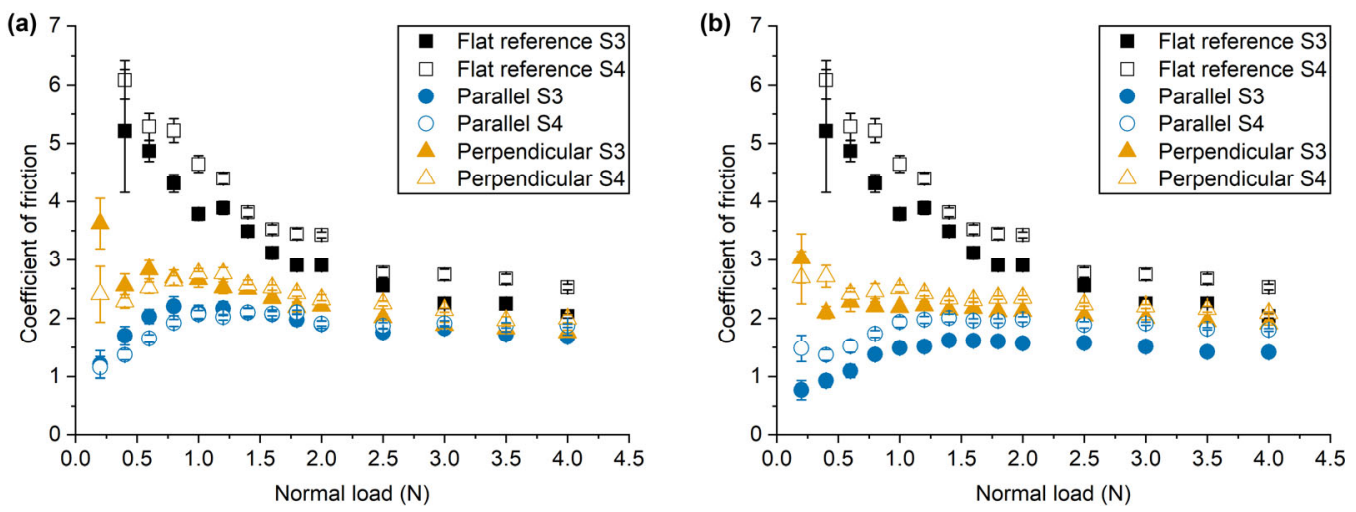

Fig. 6 Coefficient of friction for $50 \mu \mathrm{m}$ height textures (a) E100 and (b) E150. Corresponding measurement series are denoted as S3 and S4.

reduction of friction, e.g., peak values reduced from 2.7 to 2.2 and from 2.3 to 2.0 for the E100 and E150, respectively. The deformation component of friction positively correlates with deformation, which contradicts the observed behaviour and, therefore, this effect is considered insignificant. Tomlinson et al. [11] reported a considerable contribution to measured friction due to interlocking of the fingerprint ridges during sliding against the triangular-ridged textures with comparable feature dimensions produced on metals. While the interlocking effect can be responsible for the increase of friction with a change of direction, its effect is expected to reach its maximum at full contact with the textures and remain stable through the rest of the normal loads. On the contrary, the directional friction difference reduces upon reaching the full contact state with the textures, suggesting another mechanism responsible for this behaviour.

Contact area with a change of direction can be increased in two possible cases: junction growth and deformation of asperity due to shear. Skin has viscoelastic properties, which implies that during sliding over asperities a junction front can be formed. With the change of sliding direction, the junction contact area increases leading to a higher friction value. In this case, an increase of ellipsoid major radius would lead to an increase of the contact area. Contrary to that, the experimental results show lower or comparable coefficient of friction for the longer asperities.

Alternatively, a contact area rise can be the result of elastic asperity deformation due to shear stresses. Resistance to shear is proportional to the lateral cross section of the asperity. Therefore, an increase of ellipsoid major radius will increase the force required to deform the asperity. Brörmann et al. [32] performed friction measurements with cylindrical micro-pillar textures. Each pillar had a diameter and height of 50 and $20 \mu \mathrm{m}$, respectively. They observed elastic deformation of the asperities due to shear and change of contact area accompanied by a stick-slip phenomena.

\subsection{Modelling results}

The difference between parallel and perpendicular sliding directions was analysed with finite element simulations. A series of calculations was performed for each ellipsoidal asperity size. Normal displacement was changed iteratively from 5 to $40 \mu \mathrm{m}$ with a step of $5 \mu \mathrm{m}$.

For elliptic structures with the height of $30 \mu \mathrm{m}$, change of sliding direction did not affect the results. Both contact area and normal loads remained similar. However, for the asperities of $50 \mu \mathrm{m}$ height the deformation with the change of sliding direction became evident. Deformed geometry of the ellipsoid at the end of each modelling step is shown in Fig. 7. Increased asperity height under interfacial shear forces promoted an elastic deflection, which changed the contact state during tangential sliding.

Figure 8 compares development of the normal load and contact area between sliding directions for E150 $\mathrm{H} 30$ and E150 H50 at the same normal displacement of $25 \mu \mathrm{m}$. For the parallel sliding case there is a slight increase of normal load at the start of the tangential translation step. However, it returns to the initial 

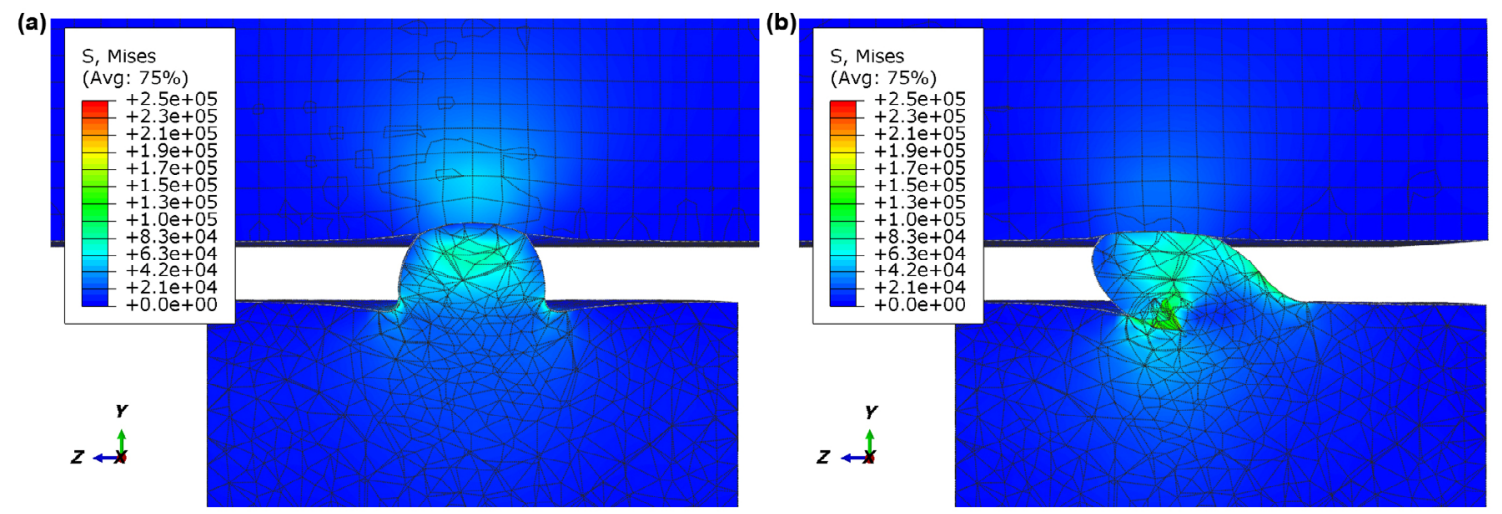

Fig. 7 Modelled deformation and stresses for E150 H50 at $30 \mu \mathrm{m}$ normal displacement: at the end of the (a) normal and (b) tangential translation steps. Perpendicular sliding, mid-section view.
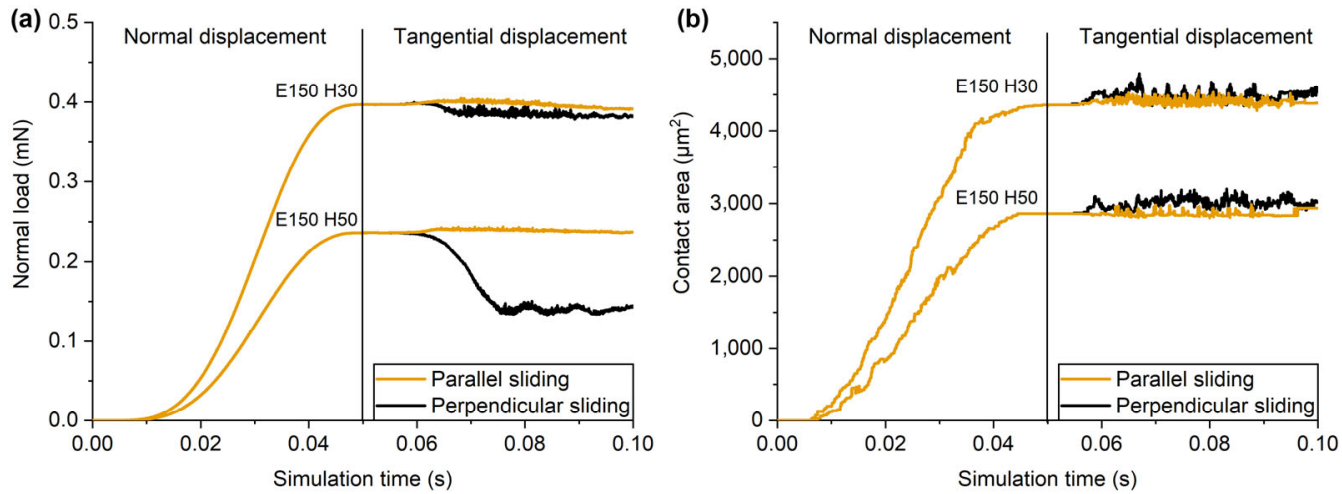

Fig. 8 (a) Normal load and (b) contact area during numerical simulation for E150 H300 and E150 H50 at $25 \mu$ m normal displacement.

value. Its calculated contact area also remains stable. Change of the sliding direction and resulted asperity deformation cause the reduction of the normal load, where it stabilised for the rest of the motion. This change is negligible for smaller asperity but is significant at asperity height of $50 \mu \mathrm{m}$ with a drop from 0.24 to $0.14 \mathrm{mN}$. It also led to fluctuations and a gradual increase of the contact area.

The data of the stable region during tangential sliding was averaged for each successful simulation run to represent the contact area as a function of normal load and is shown in Fig. 9. At low normal loads there is no directional difference in contact area. However, as the total shear force increases with the contact area, the asperity starts to bend. It leads to substantially higher contact areas and displacement with respect to the normal load. For longer asperities, the contact area nearly doubles at the same normal load as shown in Fig. 9(b).

Effectively, the asperities in the deformed state can be considered as a different texture with a lower asperity height and larger equivalent radius. Therefore, it results not only in a higher friction at asperity contact state, but the transition to the full contact state also starts at lower normal loads. As expected, an asperity with a larger equivalent radius E150 can support higher normal load before contact transition starts.

A parametric sweep was performed to qualitatively assess the influence of material elastic modulus on the deformation. Figure 10 shows that material Young's modulus significantly affects the shear force required for the deformation to occur. The relative contact area difference between sliding directions is also reduced with increase of elastic modulus, implying that a higher directional effect can be achieved with compliant materials. The maximum contact area with an asperity is limited by the transition to the full contact state, which is directly related to the tangential force. Once the full contact state is reached, a further increase of contact area for asperity becomes negligible and, therefore, the directional difference is less likely to be observed. 

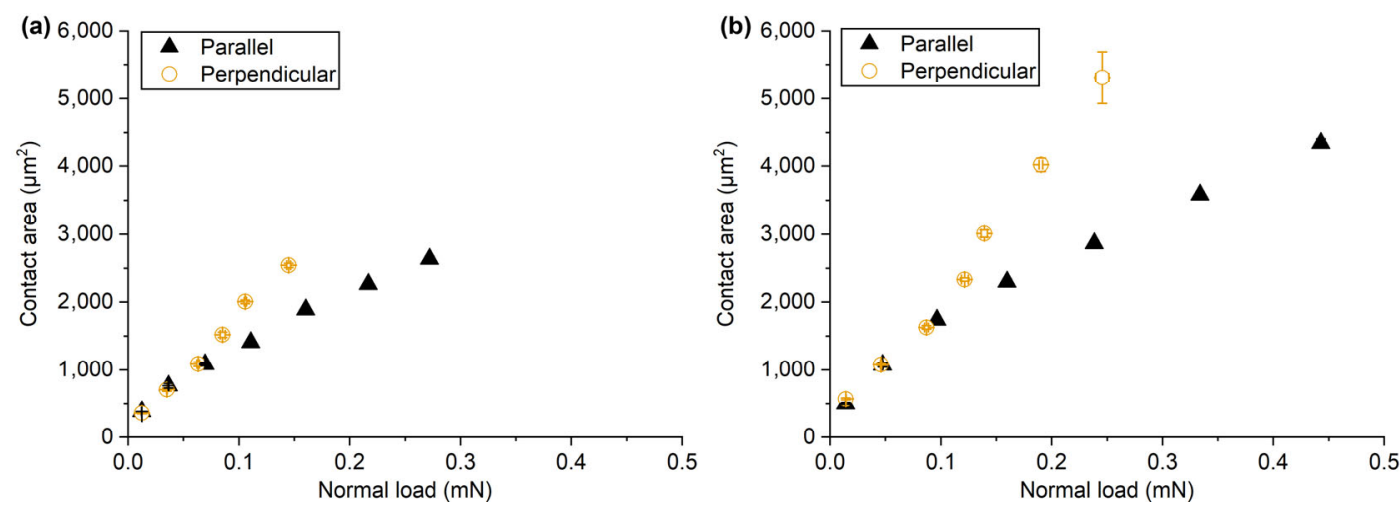

Fig. 9 Simulated contact area as a function of normal load for (a) E100 and (b) E150. Error bars represent the standard deviation due to fluctuations during sliding.

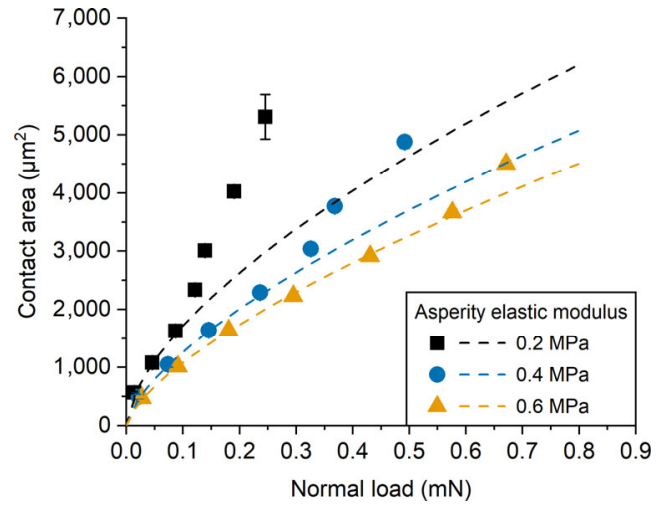

Fig. 10 Influence of asperity elastic modulus on deformation. Dashed lines show the best fits for parallel sliding, while points represent simulation results for perpendicular sliding direction.

Computational results are in a good agreement with experimental measurements. Asperity deflection explains the principal mechanism responsible for the friction gain with the change of the sliding direction. Estimated increase in contact area for E100 and E150 approaches the factors of 1.5 and 2, respectively, which matches the relationship between friction coefficients for these textures. Furthermore, the maximum difference is reached at asperity contact state before the transition to full contact, which correspond to the low normal load region on the friction graphs in Fig. 6.

\section{Conclusions}

1) Change of the sliding direction against ellipsoidal asperity textures increased the friction coefficient up to a factor of 2 . The highest difference was measured for the texture with the major radius and height of 75 and $50 \mu \mathrm{m}$, respectively.
2) Observed distinctions in tactile friction between sliding directions had predominantly adhesive nature and became the consequence of the contact area change. During perpendicular sliding, the contact area was increased due to asperity elastic deflection.

3) The highest difference between parallel and perpendicular sliding directions is predicted during asperity contact before the transition to full contact state happens. The effect increases with asperity major radius and height.

4) Finite element method (FEM) simulations show a significant influence of material elastic modulus on the normal load at which the asperity deformation starts. In the absence of asperity deformation, friction differences due to sliding direction are expected to depend on skin properties, such as its tangential stiffness and viscoelastic response.

\section{Acknowledgements}

This work was supported by INTERREG V-A Deutschland-Nederland program MOVERO under the project number 142091.

Open Access This article is licensed under a Creative Commons Attribution 4.0 International License, which permits use, sharing, adaptation, distribution and reproduction in any medium or format, as long as you give appropriate credit to the original author(s) and the source, provide a link to the Creative Commons licence, and indicate if changes were made.

The images or other third party material in this article are included in the article's Creative Commons 
licence, unless indicated otherwise in a credit line to the material. If material is not included in the article's Creative Commons licence and your intended use is not permitted by statutory regulation or exceeds the permitted use, you will need to obtain permission directly from the copyright holder.

To view a copy of this licence, visit http://creativecommons.org/licenses/by/4.0/.

\section{References}

[1] Holliins M, Faldowski R, Rao S, Young F. Perceptual dimensions of tactile surface texture: A multidimensional scaling analysis. Percept Psychophys 54(6): 697-705 (1993)

[2] Hollins M, Bensmaïa S, Karlof K, Young F. Individual differences in perceptual space for tactile textures: Evidence from multidimensional scaling. Percept Psychophys 62: 1534-1544 (2000)

[3] Skedung L, Danerlöv K, Olofsson U, Michael Johannesson C, Aikala M, Kettle J, Arvidsson M, Berglund B, Rutland M W. Tactile perception: Finger friction, surface roughness and perceived coarseness, Tribol Int 44: 505-512 (2011)

[4] Grierson L E M, Carnahan H. Manual exploration and the perception of slipperiness. Percept Psychophys 68: 1070-1081 (2006)

[5] Ndengue J D, Cesini I, Faucheu J, Chatelet E, Zahouani H, Delafosse D, Massi F. Tactile perception and friction-induced vibrations: Discrimination of similarly patterned wood-like surfaces. IEEE Trans Haptics 10: 409-417 (2017)

[6] Massimiani V, Weiland B, Chatelet E, Cornuault P H, Faucheu J, Massi F. The role of mechanical stimuli on hedonistic and topographical discrimination of textures. Tribol Int 143: 106082 (2020)

[7] Faucheu J, Weiland B, Juganaru-Mathieu M, Witt A, Cornuault P H. Tactile aesthetics: Textures that we like or hate to touch. Acta Psychol 201: 102950 (2019)

[8] Johnson S A, Gorman D M, Adams M J, Briscoe B J. The friction and lubrication of human stratum corneum. Tribol Ser 25: 663-672 (1993)

[9] Van Der Heide E, Zeng X, Masen M A. Skin tribology: Science friction? Friction 1(2): 130-142 (2013)

[10] Derler S, Gerhardt L C, Lenz A, Bertaux E, Hadad M. Friction of human skin against smooth and rough glass as a function of the contact pressure. Tribol Int 42: 1565-1574 (2009)

[11] Tomlinson S E, Carré M J, Lewis R, Franklin S E, Human finger contact with small, triangular ridged surfaces. Wear 271: 2346-2353 (2011)
[12] Darden M A, Schwartz C J. Skin tribology phenomena associated with reading braille print: The influence of cell patterns and skin behavior on coefficient of friction. Wear 332-333: 734-741 (2015)

[13] Adams M J, Briscoe B J, Johnson S A. Friction and lubrication of human skin. Tribol Lett 26: 239-253 (2007)

[14] Tomlinson S E, Lewis R, Carré M J, Franklin S E. Human finger friction in contacts with ridged surfaces. Wear 301: 330-337 (2013)

[15] Janko M, Wiertlewski M, Visell Y. Contact geometry and mechanics predict friction forces during tactile surface exploration. Sci Rep 8: 1-10 (2018)

[16] Masen M A. A systems based experimental approach to tactile friction. J Mech Behav Biomed Mater 4: 1620-1626 (2011)

[17] Tomlinson S E, Lewis R, Carré M J. The effect of normal force and roughness on friction in human finger contact Wear 267: 1311-1318 (2009)

[18] Van Kuilenburg J, Masen M A. van der Heide E. The role of the skin microrelief in the contact behaviour of human skin: Contact between the human finger and regular surface textures. Tribol Int 65: 81-90 (2013)

[19] Zhang S, Zeng X, Matthews D T A, Igartua A, RodriguezVidal E, Fortes J C, Van Der Heide E. Texture design for light touch perception. Biosurface and Biotribology 3: 25-34 (2017)

[20] Zhang S, Zeng X, Matthews D T A, Igartua A, RodriguezVidal E, Contreras Fortes J, Saenz de Viteri V, Pagano F, Wadman B, Wiklund E D, Van Der Heide E. Selection of micro-fabrication techniques on stainless steel sheet for skin friction. Friction 4(2): 89-104 (2016)

[21] Zhang S, Zeng X, Matthews D T A, Igartua A, RodriguezVidal E,. Contreras Fortes J, Van Der Heide E. Finger pad friction and tactile perception of laser treated, stamped and cold rolled micro-structured stainless steel sheet surfaces. Friction 5(2): 207-218 (2017)

[22] Duvefelt K, Olofsson U, Johannesson C M, Skedung L. Model for contact between finger and sinusoidal plane to evaluate adhesion and deformation component of friction. Tribol Int 96: 389-394 (2016)

[23] Rodríguez Urribarrí A C, Van Der Heide E, Zeng X, de Rooij M B. Modelling the static contact between a fingertip and a rigid wavy surface. Tribol Int 102: 114-124 (2016)

[24] Zhang S, Rodriguez Urribarri A, Morales Hurtado M, Zeng X, Van Der Heide E. The role of the sliding direction against a grooved channel texture on tool steel: An experimental study on tactile friction. Int $J$ Solids Struct 56: 53-61 (2015) 
[25] Darden M A, Schwartz C J. Investigation of friction mechanisms during the sliding of elastomers against hard parallel-ridge textures. Tribol Int 63: 2-7 (2013)

[26] Ji Z, Yan C, Ma S, Gorb S, Jia X, Yu B, Wang X, Zhou F. 3D printing of bioinspired topographically oriented surfaces with frictional anisotropy for directional driving. Tribol Int 132: 99-107 (2019)

[27] Wen L, Weaver J C, Lauder G V. Biomimetic shark skin: Design, fabrication and hydrodynamic function. J Exp Biol 217: 1656-1666 (2014)

[28] Klaassen M, Schipper D J, Masen M A. Influence of the relative humidity and the temperature on the in-vivo friction behaviour of human skin. Biotribology 6: 21-28 (2016)

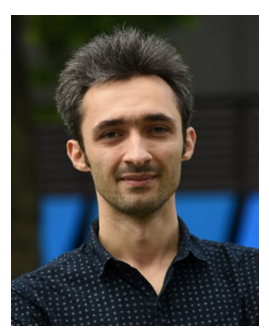

Dmitrii SERGACHEV. He obtained his joint M.Sc. degree in tribology of surfaces and interfaces from University of Coimbra, Portugal and University of Ljubljana, Slovenia in

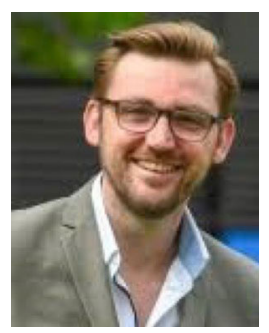

David MATTHEWS. He received his Ph.D. degree in applied physics and mathematics from the University of Groningen, the Netherlands, in 2008. Following nine years in industry as a principal researcher at Tata

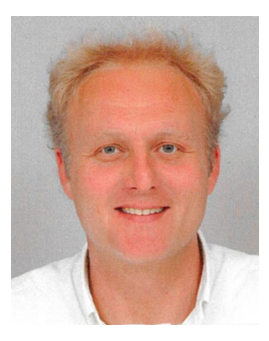

Emile VAN DER HEIDE. He obtained his M.Sc. and Ph.D. degrees in mechanical engineering from University of Twente in 1995 and 2002, respectively. His current position is a professor, chair of Skin Tribology, Laboratory for Surface
[29] Sergachev D A, Matthews D T A, Van Der Heide E. An empirical approach for the determination of skin elasticity: Finger pad friction against textured surfaces. Biotribology 18: 100097 (2019)

[30] Fagiani R, Barbieri M. A contact mechanics interpretation of the duplex theory of tactile texture perception. Tribol Int 101: 49-58 (2016)

[31] Ilg J, Rupitsch S J, Sutor A, Lerch R. Determination of dynamic material properties of silicone rubber using one-point measurements and finite element simulations. IEEE Trans Instrum Meas 61: 3031-3038 (2012)

[32] Brörmann K, Barel I, Urbakh M, Bennewitz R. Friction on a microstructured elastomer surface. Tribol Lett 50: 3-15 (2013)

2015. Currently, he is completing his Ph.D. and working as a postdoctoral researcher in the Laboratory for Surface Technology and Tribology, University of Twente. His research interests are surface texturing, contact mechanics, and skin friction.

Steel, he is now an associate professor in surface Design and engineering at the University of Twente, the Netherlands. His research interests surround the design and manufacture of surfaces for a wide range of applications and environments.

Technology and Tribology, Faculty of Engineering Technology, University of Twente. He has received President's International Fellowship 2016/2017 from Chinese Academy of Sciences. His research areas cover the surface engineering, bio-tribology, and contact mechanics. 